\title{
Influência da nutrição mineral foliar sobre doenças da parte aérea da cultura do trigo
}

\author{
Rafael Gustavo Ferreira Morales ${ }^{1}$; Idalmir dos Santos², Vanessa Nataline Tomazeli ${ }^{3}$
}

\section{RESUMO}

A utilização de fungicida para o controle de doenças foliares na cultura do trigo é prática comum entre os triticultores. Contudo, devido ao impacto ambiental provocado pelo seu uso generalizado, buscam-se alternativas para controle dos fitopatógenos. Sendo assim, este trabalho teve como objetivo avaliar o efeito da nutrição mineral foliar sobre as doenças foliares do trigo, bem como seu efeito sobre o desenvolvimento da planta. O delineamento experimental foi em blocos casualizados com quatro repetições e quatro tratamentos: 1- testemunha; 2- adubação foliar aos 30 dias após a emergência (DAE) e aos 75 DAE; 3- fungicida aos 30 e 75 DAE; e 4- adubação foliar aos 30 DAE e adubação foliar mais fungicida aos 75 DAE. Foram realizadas nove avaliações da incidência e da severidade do oídio, cujos dados foram utilizados para o cálculo das áreas abaixo das curvas de progresso da doença; três avaliações da severidade da ferrugem e da mancha da gluma; e duas avaliações da mancha amarela. Foram determinadas a massa de matéria fresca e seca da parte aérea e do sistema radicular e a produtividade da cultura. Foi observada redução da incidência e da severidade de oídio em função da adubação foliar, apenas na primeira avaliação de incidência e nas segunda e quinta avaliações de severidade da doença. O efeito complementar dos nutrientes com o fungicida para redução das doenças foi observado apenas para a mancha amarela, com redução de aproximadamente 11,16 \% da severidade. Os maiores valores de produtividade foram observados para os tratamentos apenas com fungicida e, com fungicida mais nutrientes. A adubação foliar proporcionou aumento da massa fresca de raízes e massa seca de raízes. Apesar disso, não houve aumento da massa fresca da parte aérea em função da adubação foliar.

Palavras-chave: Triticum aestivum L., indução de resistência, oídio, ferrugem, mancha amarela.

\section{ABSTRACT}

\section{Influence of leaf mineral nutrition on wheat diseases}

The use of fungicide for controlling of wheat foliar diseases is a common practice among farmers. However, due to the environmental impact caused by widespread use, some alternative pathogen controls are needed. This work aimed to evaluate the effect of leaf mineral nutrition on the control of wheat foliar diseases, and its effect on the plant. The experiment was arranged in a randomized block design with four replications and four treatments: 1- control; 2- leaf fertilization at 30 days after emergence (DAE) and at 75 DAE; 3 - fungicide at 30 and 75 DAE; and 4- leaf fertilization at $30 \mathrm{DAE}$ and foliar fertilizing + fungicide at 75 DAE. Nine incidence evaluations were carried out for powdery mildew to calculate the area under the disease progress curve; three evaluations for rust severity and gluma spots; and two evaluations for yellow spot yield, mass of fresh and dry matter of aerial parts and root system were determined. There was reduction in the intensity of powdery mildew as a function of leaf fertilization, only in the first evaluation of

\footnotetext{
Recebido para publicação em 19/10/2010 e aprovado em 31/01/2012

${ }^{1}$ Engenheiro-Agrônomo, Mestre. Universidade Federal de Lavras. Campus da UFLA, Centro, Caixa Postal 3037, 37200-000, Lavras, Minas Gerais, Brasil. moralescefet@ yahoo.com.br *Autor para correspondência.

²Engenheiro-Agrônomo. Departamento de Fitopatologia, Universidade Tecnológica Federal do Paraná, Campus Pato Branco, Via do Conhecimento, Km 01, 85503-390, Pato Branco, Paraná, Brasil. idalmir@utfpr.edu.br

${ }^{3}$ Engenheira-Agrônoma. Departamento de Fitopatologia, Universidade Tecnológica Federal do Paraná, Campus Pato Branco, Via do Conhecimento, Km 01, 85503-390, Pato Branco, Paraná, Brasil. vanetomazeli@hotmail.com
} 
incidence and in the second and fifth evaluations of severity. There was complementary effect of the nutrients with the fungicide for reducing diseases only for yellow spot, with a reduction in severity of approximately $11.16 \%$. Higher yield was recorded for the treatments with fungicide only and for fungicide + nutrients. Leaf fertilization promoted increase in root fresh mass and root dry mass; however, there was no increase in fresh mass of aerial part as a function of the leaf fertilization.

Key words: Triticum aestivum L., resistence induction, powdery mildew, rust, yellow spot.

\section{INTRODUÇÃO}

Fatores bióticos e abióticos interferem na produtividade de grãos de trigo (Triticum aestivum $\mathrm{L}$.), limitando o seu cultivo em diversas regiões do mundo. Dentre os fatores bióticos, as doenças são determinantes na redução da produtividade, aumentando consideravelmente o custo de produção por causa do maior uso de insumos. As principais doenças foliares são o oídio [Blumeria graminis (DC.) Speer], a ferrugem da folha (Puccinia triticina Erikss.), a mancha amarela [Drechslera tritici-repentis (Died.) Shoemaker], a septoriose [Mycosphaerella graminicola (Fuckel) J. Schröt.] e a mancha marrom [Bipolaris sorokiniana (Sacc.) Shoemaker] (Casa et al., 2002; Bancal et al., 2007; Serrago et al., 2009).

Entre as principais medidas de controle dessas doenças, a preferência é pelo uso de cultivares resistentes (Zhang et al., 2007). No entanto, como não se dispõem de cultivares resistentes a todas as doenças, outras medidas devem ser adotadas, como a rotação de culturas, eliminação de plantas voluntárias e hospedeiras secundárias, tratamento de sementes, aplicação de fungicidas na parte aérea e adubação equilibrada (Tripathi \& Dubey, 2004).

A aplicação de fungicidas é a principal maneira de controle das doenças foliares, sendo rotineiramente empregada nas principais regiões agrícolas do mundo. Em decorrência do impacto ambiental provocado pelo seu uso generalizado e a necessidade de conservação dos recursos naturais, buscam-se alternativas para controle desses fitopatógenos. A indução de resistência por meio da nutrição mineral pode integrar as estratégias de controles desses fitopatógenos, proporcionando a redução de custos e melhoria do ambiente.

Estudos comprovam a menor incidência de doenças em virtude de adequada nutrição mineral da planta. A redução da severidade do oídio em videira foi associada ao aumento dos níveis de N, P e K (Reuveni et al., 1993), o retardamento do desenvolvimento de míldio na cebola foi associado ao potássio (Develash \& Sugha, 1997), a incidência de cercospora em plantas de café reduziu, com o aumento da adubação de $\mathrm{K}$ e Ca (Garcia Junior et al., 2003), a severidade de ferrugem asiática (Balardin et al.,
2006) e a incidência de Phomopsis phaseoli na soja (Ito et al., 1994) foram reduzidas graças à adubação com $\mathrm{P} \mathrm{e} \mathrm{K}$. Na cultura do trigo, a aplicação foliar de cloreto de potássio foi associada com a redução de algumas manchas foliares, como a mancha da gluma (Kettlewell et al., 1990), septoriose e oídio (Cook et al., 1993).

Existe a necessidade de se conhecer até que ponto o balanço nutricional interfere na indução de resistência a doenças e como isso reflete na produtividade. Este trabalho teve como objetivo avaliar o efeito da nutrição mineral foliar sobre as doenças foliares do trigo e o seu efeito sobre o desenvolvimento da planta.

\section{MATERIAL E MÉTODOS}

O trabalho foi desenvolvido no município de Pato Branco, Paraná, Brasil, latitude $26^{\circ} 11^{\prime} 50^{\prime \prime} \mathrm{S}$, longitude $52^{\circ} 41^{\prime} 26^{\prime \prime} \mathrm{W}$ e altitude de $816 \mathrm{~m}$, clima subtropical úmido (Cfa - Koppen), sob um Latossolo Vermelho distrófico, caracterizado como profundo e de textura argilosa. A fertilidade do solo era mediana para os padrões do Estado do Paraná (Tabela 1), representando a realidade dos triticultores da região.

A semeadura foi realizada em 11 de maio, com o cultivar CD 104. As parcelas mediam $7 \mathrm{~m} \times 3 \mathrm{~m}$, com espaçamento entre linha de $20 \mathrm{~cm}$ e $0,8 \mathrm{~cm}$ entre plantas. As sementes foram tratadas com Thiram ${ }^{\circledast}(210 \mathrm{~g}$ de i.a./ $100 \mathrm{~kg}$ de sementes). A adubação de base foi realizada conforme a análise de solo, antes da semeadura, com 200 $\mathrm{kg} \mathrm{ha}^{-1}$ do formulado NPK 03-23-23, sendo a produtividade esperada de $3.000 \mathrm{~kg} \mathrm{ha}^{-1}$. Uma adubação de cobertura com $100 \mathrm{~kg} \cdot \mathrm{ha}^{-1}$ do formulado NPK 30-00-10 foi feita em todos os tratamentos no início do perfilhamento, seguindo as recomendações utilizadas no Estado do Paraná (Comissão de Química e Fertilidade do Solo-RS/SC, 2004).

O delineamento experimental foi em blocos casualizados com quatro tratamentos e quatro repetições. Os nutrientes foram aplicados utilizando produtos comerciais, próprios para a cultura, formulados em dois pacotes $(\mathrm{P})$ : P1 [N (7\%), P (15\%), K (15\%), S (5\%), B (2\%), Cu (1\%), Mn (5\%), Mo $(0,06 \%)$ e Zn (3,5\%)]; e P2 [K (30\%), B (2\%) e Ca $(10 \%)]$. Com base nessas formulações, foram realizados 
os seguintes tratamentos: 1- testemunha (água); 2- P1 (1,5 $\left.\mathrm{kg} \mathrm{ha}^{-1}\right)$ aos 30 dias após a emergência (DAE) e P2 (2,0 kg ha $^{-1}$ ) aos 75 DAE (grão leitoso); 3- fungicida (pyraclostrobin + epoxiconazole $)\left(80+40\right.$ g i.a. ha $\left.{ }^{-1}\right)$ aos 30 e 75 DAE; e 4- P1 (1,5 kg ha $\left.{ }^{-1}\right)$ aos 30 DAE e P2 $\left(2,0 \mathrm{~kg} \mathrm{ha}^{-1}\right)$ mais fungicida (pyraclostrobin + epoxiconazole) $(80+40 \mathrm{~g}$ i.a. $\left.\mathrm{ha}^{-1}\right)$ aos 75 DAE.

Os fungicidas foram aplicados com pulverizador costal propelido à $\mathrm{CO}_{2}$, com barra munida de bicos tipo leque 110-02, espaçados em $0,50 \mathrm{~m}$, regulado para aplicar um volume de calda de $200 \mathrm{~L} \mathrm{ha}^{-1}$.

Antes da aplicação dos tratamentos, foram realizadas as avaliações da incidência e severidade do oídio para confirmar a homogeneidade da doença (pré-spray). Após a aplicação dos primeiros tratamentos, aos $30 \mathrm{DAE}$, realizaram-se as avaliações semanais da incidência (percentagem de folhas infectadas em relação ao total) e da severidade (percentagem da área foliar coberta com sintomas) do oídio, para isso, utilizou-se escala diagramática (Reis et al., 1979). As avaliações foram realizadas em laboratório, com 20 plantas de cada parcela coletadas ao acaso, descartando as folhas senescentes e que não estavam completamente desenvolvidas. As avaliações foram semanais, até a redução acentuada dos sintomas da doença, coincidindo com o início das avaliações das doenças de final de ciclo. Foram realizadas três avaliações semanais da ferrugem, da mancha amarela e da mancha da gluma, conforme escala de nota própria para cada doença (Saari \& Prescott, 1975), utilizando-se 20 plantas por parcela.

A incidência e a severidade do oídio também foram avaliadas pela área abaixo da curva de progresso da doença (AACPD), com base nos dados da incidência e da severidade das nove avaliações, utilizando-se a fórmu1a: $A A C P D=\sum_{i}^{n-1}\left(\frac{y_{i}+y_{i+1}}{2}\right)\left(t_{i+1}-t_{i}\right)($ Shaner \& Finney, 1977), em que ( $n$ ) é o número de avaliações, (y) é a intensidade da doença e $\left(t_{i+1}-t_{i}\right)$ é o intervalo em dias entre duas avaliações consecutivas.

Aos 100 DAE, foi determinada a massa de matéria fresca e seca da parte aérea e do sistema radicular de 10 plantas por parcela. Para massa de matéria seca, utilizou-se estufa de secagem a $70{ }^{\circ} \mathrm{C}$, até peso constante das amostras (4 dias).
Em virtude das avaliações destrutivas, foi determinado o estande após a implantação da cultura e antes da análise do rendimento, contando-se o número de plantas em três fileiras centrais de cada parcela. Os valores de produtividade foram ajustados levando em consideração o estande inicial. A produtividade foi determinada nas cinco linhas centrais de cada parcela, descartando-se as laterais por efeito de bordadura, utilizando-se a colhedora de parcelas marca Wintersteiger. Após a colheita, os grãos passaram pelos processos de secagem, limpeza e pesagem, determinando-se o peso de 1.000 sementes.

Os dados da incidência, da severidade das doenças, da AACPD, da massa de matéria fresca e seca da raiz e da parte aérea, do rendimento e o do peso de mil sementes foram submetidos à análise de variância e as médias comparadas pelo teste de Tukey $(\mathrm{p}<0,05)$.

\section{RESULTADOS E DISCUSSÃO}

$\mathrm{Na}$ avaliação inicial, a incidência e a severidade do oídio eram homogêneas na área experimental, antes da aplicação dos tratamentos (Tabela 2). Foi observada redução da incidência e da severidade de oídio em função da adubação foliar, apenas na primeira avaliação de incidência e, na segunda e quinta avaliações de severidade da doença. A principal diferença observada foi nos tratamentos com fungicida e fungicida mais nutrientes, com redução da incidência até a quarta avaliação e da severidade até a quinta avaliação. A partir dessa fase, a doença ficou homogênea em todo o experimento, voltando a existir diferenças na incidência apenas na oitava avaliação, após as aplicações aos 75 DAE. Na AACPD, que sumariza as avaliações individuais da incidência e da severidade em um único valor, não foi observada redução significativa em relação à testemunha proporcionada pelas aplicações dos nutrientes. Contudo, houve redução proporcionada pelos tratamentos com fungicida e fungicida mais nutrientes, sem diferença entre estes (Tabela 2). Para severidade da ferrugem, similarmente a severidade de oídio, não houve diferença entre o tratamento com nutrientes (sem fungicida) e a testemunha. O mesmo foi observado para os dois tratamentos que continham fungicida, que apresentaram menor severidade e não diferiram entre si (Tabela 3).

Tabela 1. Resultado da análise química do solo da área experimental, referente à camada de 0 a $20 \mathrm{~cm}$

\begin{tabular}{|c|c|c|c|c|c|c|}
\hline pH & C-orgânico & $\mathrm{Ca}$ & Mg & $\mathbf{K}$ & $\mathbf{H}+\mathbf{A l}$ & Al \\
\hline$\left(\mathrm{CaCl}_{2}\right)$ & $\mathrm{g} \mathrm{dm}^{-3}$ & \multicolumn{5}{|c|}{ cmolc dm ${ }^{-3}$} \\
\hline 4,6 & 14 & 3,10 & 0,70 & 0,15 & 2,20 & 0,90 \\
\hline P (Mehlich) & $\mathrm{S}-S O_{4}^{-2}$ & B & $\mathrm{Cu}$ & $\mathrm{Fe}$ & Mn & $\mathbf{Z n}$ \\
\hline \multicolumn{7}{|c|}{$\mathrm{mg} \mathrm{kg}^{-1}$} \\
\hline 7,00 & 4,00 & 0,55 & 12,40 & 19,00 & 53,00 & 10,10 \\
\hline
\end{tabular}




\begin{tabular}{|c|c|c|c|c|c|c|c|c|c|c|c|c|c|c|c|c|c|c|}
\hline \multirow{5}{*}{ 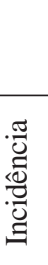 } & \multirow{5}{*}{$\begin{array}{l}\text { Tratamentos } \\
\text { Testemunha } \\
\text { Nutrientes } \\
\text { Fungicida } \\
\text { Nutri + Fung }\end{array}$} & \multirow{2}{*}{$\begin{array}{c}\begin{array}{c}\text { Pré-spray } \\
\text { 1/jul }\end{array} \\
63,38^{*}\end{array}$} & \multicolumn{2}{|c|}{$\begin{array}{l}1^{a} A v . \\
9 / j u l\end{array}$} & \multicolumn{2}{|c|}{$\begin{array}{l}2^{\mathrm{a}} \mathrm{Av} . \\
20 / \mathrm{jul}\end{array}$} & \multicolumn{2}{|c|}{$\begin{array}{l}3^{\text {a Av. }} \\
\text { 29/jul }\end{array}$} & \multicolumn{2}{|c|}{$\begin{array}{l}4^{\mathrm{a} A v .} \\
\text { 7/ago1 }\end{array}$} & \multicolumn{2}{|l|}{$\begin{array}{l}5^{\text {a Av }} \\
\text { 4/ago }\end{array}$} & \multirow{2}{*}{$\begin{array}{c}\begin{array}{c}6^{\text {a }} \text { Av. } \\
\text { 20/ago }\end{array} \\
60,31^{*}\end{array}$} & \multirow{2}{*}{$\begin{array}{l}7^{\text {a Av. }} \\
\text { 27/ago }\end{array}$} & $\begin{array}{l}8^{\mathrm{a}} \mathrm{Av} \\
10 / \mathrm{set}\end{array}$ & \multicolumn{2}{|c|}{$\begin{array}{c}9^{\text {a }} \text { Av. } \\
\text { 20/set }\end{array}$} & AACPD \\
\hline & & & 68,50 & $\mathrm{a}$ & 64,37 & $\mathrm{a}$ & 43,32 & $\mathrm{a}$ & 62,43 & $\mathrm{a}$ & $55,88^{*}$ & & & & 39,29 a & 13,58 & $\mathrm{a}$ & 3761,73 \\
\hline & & 63,79 & 54,47 & $\mathrm{~b}$ & 59,66 & $\mathrm{a}$ & 39,72 & $\mathrm{a}$ & 60,05 & $\mathrm{a}$ & 49,98 & & 58,62 & 52,80 & $33,45 \mathrm{ab}$ & 17,66 & $\mathrm{a}$ & 3513,11 a \\
\hline & & 64,19 & 15,57 & $\mathrm{c}$ & 44,11 & $\mathrm{~b}$ & 35,33 & $a b$ & 37,80 & $\mathrm{~b}$ & 44,69 & & 60,69 & 54,99 & $25,84 \mathrm{bc}$ & 0,51 & $\mathrm{~b}$ & $2803,41 \mathrm{~b}$ \\
\hline & & 65,09 & 7,47 & $\mathrm{~d}$ & 41,45 & $\mathrm{~b}$ & 25,98 & $\mathrm{~b}$ & 37,69 & $\mathrm{~b}$ & 43,53 & & 60,21 & 53,86 & $19,10 \quad \mathrm{c}$ & 0,00 & $\mathrm{~b}$ & $2570,00 \mathrm{~b}$ \\
\hline & C. V. & 12,55 & 18,3 & & 20,28 & & 18,90 & & 17,42 & & 29,29 & & 32,12 & 20,01 & 20,67 & 13,98 & & \\
\hline \multirow{5}{*}{ 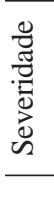 } & Testemunha & 12,13 & 15,40 & $\mathrm{a}$ & 8,26 & $\mathrm{a}$ & 6,36 & $\mathrm{a}$ & 8,32 & $\mathrm{a}$ & 10,49 & $\mathrm{a}$ & $2,59 *$ & $2,42 *$ & $0,83^{*}$ & $0,65^{*}$ & & 425,43 a \\
\hline & Nutrientes & 12,45 & 12,03 & $\mathrm{a}$ & 6,90 & $\mathrm{~b}$ & 5,21 & $\mathrm{a}$ & 4,82 & $\mathrm{a}$ & 6,03 & $\mathrm{~b}$ & 2,31 & 2,19 & 1,84 & 1,01 & & 322,38 \\
\hline & Fungicida & 11,21 & 2,94 & $\mathrm{~b}$ & 3,91 & $\mathrm{c}$ & 2,82 & $\mathrm{~b}$ & 2,62 & $\mathrm{~b}$ & 5,31 & $\mathrm{~b}$ & 1,87 & 1,36 & 0,16 & 0,11 & & $176,18 \mathrm{~b}$ \\
\hline & Nutri + Fung & 13,01 & 2,25 & $\mathrm{~b}$ & 2,62 & $\mathrm{c}$ & 2,39 & $\mathrm{~b}$ & 2,35 & $\mathrm{~b}$ & 4,20 & $\mathrm{~b}$ & 2,24 & 1,27 & 0,15 & 0,00 & & $147,11 \mathrm{~b}$ \\
\hline & C. V. & 13,73 & 8,26 & & 10,78 & & 15,83 & & 12,47 & & 16,45 & & 25,41 & 22,26 & 14,57 & 5,13 & & 20,99 \\
\hline
\end{tabular}

Médias, seguidas pela mesma letra minúscula na coluna, não diferem estatisticamente entre si pelo teste de Tukey $(\mathrm{p}<0,05)$. *diferença não significativa.

Tabela 3. Efeito da nutrição mineral foliar, da aplicação de fungicida e de ambos (nutrientes + fungicida) sobre a severidade de ferrugem (Puccinia triticina Erikss.), mancha amarela [Drechslera tritici-repentis (Died.) Shoemaker] e mancha da gluma [Mycosphaerella graminicola (Fuckel) J. Schröt.] na cultura do trigo

\begin{tabular}{|c|c|c|c|c|c|c|c|c|c|c|c|c|c|c|c|c|c|c|c|c|}
\hline \multirow{3}{*}{$\frac{\text { Tratamentos }}{\text { Testemunha }}$} & \multicolumn{8}{|c|}{ Ferrugem } & \multicolumn{5}{|c|}{ Mancha Amarela } & \multicolumn{7}{|c|}{ Mancha da Gluma } \\
\hline & \multicolumn{2}{|c|}{$\begin{array}{c}1^{\text {a Av. }} \\
20 / \text { set }\end{array}$} & \multicolumn{2}{|c|}{$\begin{array}{c}2^{\text {a }} \text { Av. } \\
\text { 27/set }\end{array}$} & \multicolumn{2}{|c|}{$\begin{array}{l}3^{a} \text { Av. } \\
\text { 4/out }\end{array}$} & \multicolumn{2}{|c|}{ AACPD } & \multirow{2}{*}{$\begin{array}{r}\mathbf{1}^{\text {a }} \mathbf{A v} . \\
\mathbf{2 0 / s e t}\end{array}$} & \multicolumn{2}{|c|}{$\begin{array}{l}2^{\mathrm{a}} \mathrm{Av} . \\
27 / \mathrm{set}\end{array}$} & \multicolumn{2}{|c|}{ AACPD } & \multicolumn{2}{|c|}{$\begin{array}{l}1^{\text {a Av. }} \\
20 / \text { set }\end{array}$} & \multicolumn{2}{|c|}{$\begin{array}{l}2^{\text {a Av. }} \\
27 / \text { set }\end{array}$} & \multicolumn{2}{|c|}{$\begin{array}{l}3^{a} \text { Av. } \\
\text { 4/out }\end{array}$} & AACPI \\
\hline & 15,28 & $\mathrm{a}$ & 17,40 & $\mathrm{a}$ & 59,69 & $\mathrm{a}$ & 524,79 & $\mathrm{a}$ & & 67,56 & $\mathrm{a}$ & 849,10 & $\mathrm{a}$ & 4,27 & $\mathrm{a}$ & 5,67 & $\mathrm{a}$ & 12,25 & a & 115,64 \\
\hline Nutrientes & 12,78 & $\mathrm{a}$ & 8,64 & $\mathrm{~b}$ & 59,58 & $\mathrm{a}$ & 506,52 & a & 55,44 & 53,11 & $a b$ & 759,85 & $\mathrm{~b}$ & 3,45 & $\mathrm{~b}$ & 4,75 & $\mathrm{~b}$ & 8,12 & $\mathrm{~b}$ & 80,99 \\
\hline Fungicida & 2,41 & $\mathrm{~b}$ & 1,80 & $\mathrm{c}$ & 8,15 & $\mathrm{~b}$ & 73,92 & $\mathrm{~b}$ & 60,21 & 51,13 & $\mathrm{~b}$ & 779,38 & $\mathrm{~b}$ & 3,59 & $\mathrm{~b}$ & 4,12 & $\mathrm{~b}$ & 5,14 & $\mathrm{bc}$ & 61,11 \\
\hline Nutri + Fung & 3,02 & $\mathrm{~b}$ & 1,04 & $\mathrm{c}$ & 8,69 & $\mathrm{~b}$ & 81,97 & $\mathrm{~b}$ & 53,20 & 45,71 & $\mathrm{~b}$ & 692,37 & $\mathrm{c}$ & 3,44 & $\mathrm{~b}$ & 4,21 & $\mathrm{~b}$ & 4,16 & $\mathrm{c}$ & 53,20 \\
\hline C. V. & 13,8 & & 19 & & 12,6 & & - & & 31,76 & 22,9 & & - & & 22 , & & 14, & & 23 & & - \\
\hline
\end{tabular}

Médias, seguidas pela mesma letra minúscula na coluna, não diferem estatisticamente entre si pelo teste de Tukey (p<0,05). *diferença não significativa 
Quanto à severidade da Mancha Amarela, o tratamento somente com nutrientes reduziu em $10,51 \%$ na AACPD em relação à testemunha, não diferindo em relação ao tratamento somente com fungicida. O efeito complementar dos nutrientes com o fungicida para redução das doenças foi observado apenas para a mancha amarela, com redução de aproximadamente $11,16 \%$ da severidade (Tabela 3). Os efeitos da nutrição mineral sobre doenças no trigo também foram observados por outros autores. A aplicação equilibrada de $\mathrm{N}, \mathrm{P}$ e K reduziu a severidade de helmintosporiose em 15 e $22 \%$ em 2001 e 2002, respectivamente (Sharma et al., 2006). Os mesmos autores observaram que tanto o $\mathrm{KCl}$ como o $\mathrm{CaCl}_{2}$ reduziram a severidade da mancha, sendo o primeiro $11 \%$ mais eficiente que o segundo. Em outro estudo, plantas bem nutridas aumentaram tanto a produtividade $(52,1 \%)$ como o peso de mil sementes (PMS) (15,5\%), sendo esses aumentos relacionados com a redução das manchas de helmintosporiose (Sharma \& Duveiller, 2004).

Entre os nutrientes, o potássio é descrito como o que exerce mais influência sobre as doenças, pois aumenta a resistência ao desenvolvimento de alguns patógenos, aumenta a espessura da parede celular, proporciona maior rigidez dos tecidos e promove a rápida recuperação após injúrias (Basseto et al., 2007). A carência de potássio também esta relacionada com a menor síntese de compostos de alto peso molecular, favorecendo o acúmulo de compostos de baixo peso molecular, propiciando o desenvolvimento de doenças (Adebitan, 1998). O cálcio é outro nutriente que possui grande relevância, pois é essencial para a estabilidade das biomembranas (Chitarra et al., 2000) e pela inibição da atividade da enzima poligalacturonase (Conway et al., 1991). Os micronutrientes, como o cobre, boro e manganês, também têm funções importantes que influenciam na síntese de lignina e fenóis simples (Ceretta $e t$ al., 2005). O principal fator a ser levado em consideração, entretanto, é a ação conjunta de todos os nutrientes, impossibilitando o desenvolvimento fúngico pela ação concomitante de vários mecanismos de inibição do patógeno.
Os maiores valores de produtividade foram observados para os tratamentos com fungicida somente e fungicida mais nutrientes (Tabela 4). A produtividade apresentou alta correlação com o PMS $(r=0,91)$. No entanto, para PMS, o tratamento com fungicida mais nutrientes foi superior ao tratamento com fungicida somente. $\mathrm{O}$ aumento da produção e da qualidade de grãos pode ser alcançado quando um fertilizante foliar é adicionado ao fungicida, como já relatado para fungicidas triazóis (Blandino \& Reyneri, 2009).

A adubação foliar proporcionou aumento da massa fresca de raízes de 14,23 para 17,34 $\mathrm{g} \mathrm{pl}^{-1}$, incremento este equivalente a $21,85 \%$. O mesmo foi observado para massa seca de raízes, com incremento de $16,56 \%$. Por outro lado, o tratamento fungicida não diferiu da testemunha, tanto para massa fresca como para massa seca (Tabela 3). Apesar de aumentar a produção de raízes, não houve aumento da massa fresca da parte aérea (MFA) em função da adubação foliar. Porém, o aumento da massa verde aérea, em função da fertilização foliar com macro e micronutrientes, foi observado por outros autores (Blandino \& Reyneri, 2009), tendo como resultado a manutenção da longevidade do dossel durante o enchimento de grãos com reflexos positivos na produtividade. Quando os nutrientes foram aplicados junto com o fungicida, entretanto, houve aumento da MFA, mostrando um efeito complementar entre os dois produtos em relação à sua aplicação isolada (Tabela 4). Esse fato pode estar relacionado com o $\mathrm{N}$ do adubo foliar, pois, quando aplicado junto com o fungicida, há aumento da sua absorção e redistribuição na planta (Gooding et al., 2007). Fato semelhante foi observado em outros trabalhos, em que foi obtido o aumento da produção de grãos e do teor de proteínas quando aplicado, na mesma calda de pulverização, o $\mathrm{N}$ e o fungicida (Dimmock \& Gooding, 2002).

Apesar de ter sido observada pouca influência da fertilização foliar sobre as doenças, cabe ressaltar que essa interação é influenciada pela cultura em estudo, tipo de solo, concentração e época de aplicação dos nutrientes, dentre outros fatores. Todavia, os resultados observados para mancha amarela indicam que a técnica é promissora.

Tabela 4. Efeito da nutrição mineral foliar, da aplicação de fungicida e de ambos (nutrientes+fungicida) sobre a produtividade, peso de mil sementes (P.M.S.), massa de matéria fresca de raízes (M.F.R.), massa de matéria seca de raízes (M.S.R.), massa de matéria fresca aérea (MFA) e massa de matéria seca aérea (M.A.S.) da cultura do trigo

\begin{tabular}{|c|c|c|c|c|c|c|c|c|c|c|c|c|}
\hline \multirow{3}{*}{$\begin{array}{l}\text { Tratamentos } \\
\text { Testemunha }\end{array}$} & \multicolumn{3}{|c|}{ Produtividade } & \multirow{2}{*}{$\begin{array}{l}\text { PMS } \\
(g)\end{array}$} & \multicolumn{2}{|c|}{ M.F.R. } & \multicolumn{2}{|c|}{ M.S.R. } & \multicolumn{2}{|c|}{ M.F.A. } & \multicolumn{2}{|c|}{ M.S.A. } \\
\hline & \multicolumn{2}{|c|}{$\left(\mathrm{t} \mathrm{ha}^{-1}\right)$} & (g) & & \multicolumn{8}{|c|}{$\left(\mathrm{g} \mathrm{pl}^{-1}\right)$} \\
\hline & 2,47 & $\mathrm{a}$ & 46,21 & $\mathrm{a}$ & 14,23 & b & 4,65 & $\mathrm{~b}$ & 39,45 & $\mathrm{a}$ & 8,55 & a \\
\hline Nutrientes & 2,77 & $a b$ & 48,78 & $a b$ & 17,34 & $\mathrm{a}$ & 5,42 & $\mathrm{a}$ & 41,75 & $\mathrm{a}$ & 8,90 & $\mathrm{a}$ \\
\hline Fungicida & 3,00 & $\mathrm{bc}$ & 48,12 & $\mathrm{~b}$ & 16,12 & $a b$ & 4,55 & $\mathrm{~b}$ & 57,30 & $\mathrm{~b}$ & 12,05 & $\mathrm{~b}$ \\
\hline Nutri + Fung & 3,26 & $\mathrm{c}$ & 51,01 & $\mathrm{c}$ & 17,01 & $\mathrm{a}$ & 5,57 & $\mathrm{a}$ & 66,25 & $\mathrm{c}$ & 12,15 & $\mathrm{~b}$ \\
\hline C.V. & 22,91 & & 10,10 & & 12,44 & & 17,90 & & 12,55 & & 12,00 & \\
\hline
\end{tabular}

Médias, seguidas pela mesma letra minúscula na coluna, não diferem estatisticamente entre si pelo teste de Tukey (p<0,05). 


\section{CONCLUSÕES}

A incidência e a severidade do oídio, a severidade da ferrugem e da mancha da gluma não foram influenciadas pela adubação foliar.

A severidade da mancha amarela reduziu em função da adubação foliar.

A massa de matéria fresca e a massa seca de raízes aumentaram devido à adubação foliar.

\section{REFERÊNCIAS}

Adebitan SA (1998) Evaluation of potassium fertilizer and tillage depth for the control of web blight of cowpea. International Journal of Tropical Plant Diseases, 16:173- 182.

Balardin RS, Dallagnol LJDHT \& Navarini L (2006) Influência do Fósforo e do Potássio na Severidade da Ferrugem da Soja Phakopsora pachyrhizi. Fitopatologia Brasileira, 31:462-467.

Bancal MO, Robert C \& Ney B (2007) Modelling wheat growth and yield losses from late epidemics of foliar diseases using loss of green area per layer and pre-anthesis reserves. Annals of Botany, 100:777-789.

Basseto MA, Ceresini PC \& Valério Filho WV (2007) Severidade da mela da soja causada por Rhizoctonia solani AG-1 IA em função de doses de potássio. Summa Phytopathologica, 33:5662.

Blandino M \& Reyneri A (2009) Effect of fungicide and foliar fertilizer application to winter wheat at anthesis on flag leaf senescence, grain yield, flour bread-making quality and DON contamination. European Journal of Agronomy, 30:275-282.

Casa RT, Hoffmann LL, Panisson E, Mendes CC \& Reis EM (2002) Sensibilidade de Blumeria graminis f.sp. tritici a alguns fungicidas. Fitopatotologia Brasileira, 27:626-630.

Ceretta CA, Pavinato A, Pavinato PS, Moreira ICL, Girotto E \& Trentin EE (2005) Micronutrientes na soja: produtividade e análise econômica. Ciência Rural, 35:576-581.

Chitarra AB, Evangelista RM \& Chitarra MIF (2000) Influência da aplicação pré-colheita de cálcio na textura e na atividade das enzimas poligalacturonase, pectinametilesterase e bgalactosidase de mangas 'Tommy Atkins' armazenadas sob refrigeração. Ciência e Agrotecnologia, 24:174-181.

Comissão de Química e Fertilidade do Solo - RS/SC (2004) Manual de adubação e calagem para os Estados do Rio Grande do Sul e de Santa Catarina. 10.ed. Porto Alegre, SBCS - Núcleo Regional Sul/UFRGS. 400p.

Conway WS, Sams CE, Abbott JA \& Bruton BD (1991) Postharvest calcium treatment of apple fruit to provide broad-spectrun protection against postharvest pathogens. Plant Disease, 75:620-622.

Cook JW, Kettlewell PS \& Parry DW (1993) Control of Erysiphe graminis and Septoria tritici on wheat with foliar-applied potassium chloride. Journal of the Science of Food and Agriculture, 63:126.

Develash RK \& Sugha S (1997) Factors affecting development of downy mildew (Peronospora destructor) of onion (Allium cepa). Indian Journal of Agricultural Sciences, 67:71-74.

Dimmock JPRE \& Gooding MJ (2002) The influence of foliar disease, and their control by fungicides, on the protein concentration in wheat grain: a review. Journal of Agriculture Science, 138:349-366.
Garcia Junior D, Pozza EA, Pozza AAA, Souza PE, Carvalho JG \& Balieiro AC (2003) Incidência e severidade da cercosporiose do cafeeiro em função do suprimento de potássio e cálcio em solução nutritiva. Fitopatologia Brasileira, 28:286-291.

Gooding MJ, Gregory PJ, Ford KE \& Ruske RE (2007) Recovery of nitrogen from different sources following applications to winter wheat at and after anthesis. Field Crops Research, 100:143-154.

Ito MF, Mascarenhs HAA, Tanaka MAS, Dudienas C, Tanaka RT, Gallo PB \& Miranda MAC (1994) Efeito residual da adubação potássica e da calagem sobre a incidência de Phomopsis spp. em sementes de soja. Fitopatologia Brasileira, 19:44-49.

Kettlewell PS, Gil B \& Domleo RL (1990) Evaluation of lateseason foliar application of potassium chloride for disease control in winter wheat. Journal of Fertilizer Issues, 7:17-23.

Reis EM, Minella E, Baier AC \& Santos HP (1979) Reação de cultivares e linhagens de trigo a Erysiphe graminis (DC) f. sp. tritici Marchall. Summa Phytopathologica, 5:52-64.

Reuveni M, Naor A, Reuveni R, Shimoni M \& Bravdo B (1993) The influence of NPK fertilization rates on susceptibility to powdery mildew of field-grown wine grapes. Journal of Small Fruit and Viticulture, 2:31-41.

Saari EE \& Prescott JM (1975) A scale for appraising the foliar intensity of winter wheat diseases. Plant Disease Reporter, 595:377-380.

Serrago RA, Carretero R, Bancal MO \& Miralles DJ (2009) Foliar diseases affect the eco-physiological attributes linked with yield and biomass in wheat (Triticum aestivum L.). European Journal of Agronomy, 31:195-203.

Shaner G \& Finney RF (1977) The effects of nitrogen fertilization on the expression of show-mildwing in knox wheat. Phytopathology, 67:1051-1055.

Sharma P, Duveiller E \& Sharma RC (2006) Effect of mineral nutrients on spot blotch severity in wheat, and associated increases in grain yield. Field Crops Research, 95:426-430.

Sharma RC \& Duveiller E (2004) Effect of helminthosporium leaf blight on performance of timely and late-seeded wheat under optimal and stressed levels of soil fertility and moisture. Field Crops Research, 89:205-218.

Tripathi P \& Dubey NK (2004) Exploitation of natural products as an alternative strategy to control postharvest fungal rotting of fruit and vegetables. Postharvest Biology and Technology, 32:235-245.

Zhang XY, Loyce C, Meynard JM \& Monod H (2007) Modeling the effect of cultivar resistance on yield losses of winter wheat in natural multiple disease conditions. European Journal of Agronomy, 26:384-393. 